\title{
Ding's herbal enema treats dextran sulfate sodium-induced colitis in mice by regulating the gut microbiota and maintaining the Treg/Th17 cell balance
}

\author{
YAN-YAN TAN $^{1 *}$, YANG DING $^{2 *}$, XUEPING ZHENG $^{1}$, GONG-JIAN DAI $^{1}$, SU-MIN ZHANG ${ }^{1}$, XU YANG $^{1}$, \\ DA-CHAO XU ${ }^{1}$, PENG CHEN ${ }^{2}$, JIA-MIN ZHANG ${ }^{2}$, JIA-ZE MA ${ }^{2},{\text { MENG } \text { LI }^{2} \text {, SHI-CAI HUANG }}^{2}$, \\ YAN LIU $^{2}$, YU-TING ZHANG ${ }^{2}$, HAN XING $^{2}$, KANG DING $^{1}$ and YI-JIANG DING ${ }^{1}$ \\ ${ }^{1}$ Department of National Center of Colorectal Surgery, Jiangsu Integrate Colorectal Oncology Center, Nanjing Hospital of
Chinese Medicine Affiliated to Nanjing University of Chinese Medicine, Nanjing, Jiangsu 210001;
${ }^{2}$ Department of First Clinical Medical College, Nanjing University of Chinese Medicine, Nanjing, Jiangsu 210029, P.R. China
}

Received July 27, 2020; Accepted August 18, 2021

DOI: $10.3892 / \mathrm{etm} .2021 .10802$

\begin{abstract}
Ding's herbal enema (DHEP) is a traditional Chinese medicinal therapy that has been used to treat ulcerative colitis (UC) in China. The present study determined the molecular mechanism of the effect of DHEP in UC treatment. C57BL/6J mice were treated with $3.5 \%(\mathrm{w} / \mathrm{v})$ dextran sulfate sodium (DSS) for 7 days to establish an animal model of colitis. The mice were divided into five groups $(n=5)$ : Control, vehicle, DHEP, mesalazine and $\beta$-sitosterol. After oral administration for 7 days, the body weight, disease activity index, histopathology and inflammatory factors were analyzed. The fractions of $\mathrm{CD}^{+}{ }^{+} \mathrm{Foxp}^{+}$regulatory $\mathrm{T}$ (Treg) cells and $\mathrm{CD} 4^{+} \mathrm{IL}-17 \mathrm{~A}^{+}$ $\mathrm{T}$ helper (Th) cells were determined by flow cytometry. Gut microbiota composition was analyzed by next-generation sequencing. The results revealed that DHEP and $\beta$-sitosterol could significantly alleviate the symptoms of DSS-induced UC. Furthermore, the levels of IL-6, cyclooxygenase-2, TNF- $\alpha$ and p65 were reduced after administration of DHEP. Additionally, the data indicated that DHEP could increase the abundance of seven operational taxonomic units (OTUs) and decrease the abundance of 12 OTUs in the gut microbiota. The content of short-chain fatty acids in the colon remodeled the balance of Treg/Th17 cells in DSS-induced UC in mice. The present study preliminarily defined the mechanism of action of
\end{abstract}

Correspondence to: Professor Kang Ding or Professor Yi-Jiang Ding, Department of National Center of Colorectal Surgery, Jiangsu Integrate Colorectal Oncology Center, Nanjing Hospital of Chinese Medicine Affiliated to Nanjing University of Chinese Medicine, 1 Jinling Road, Nanjing, Jiangsu 210001, P.R. China

E-mail: dingkang1123@163.com

E-mail:2543775235@qq.com

*Contributed equally

Key words: Ding's herbal enema, $\beta$-sitosterol, ulcerative colitis, short-chain fatty acids, $\mathrm{T}$ cell differentiation, gut microbiota
DHEP in UC that may be associated with the regulation of the gut microbiota composition, and maintenance of the balance between Treg and Th17 cells. Furthermore, $\beta$-sitosterol exhibited the same effects with DHEP and it could be a possible substitute for DHEP in UC treatment.

\section{Introduction}

Inflammatory bowel disease (IBD), including ulcerative colitis (UC) and Crohn's disease, is an autoimmune disease of the intestine (1). IBD is influenced by a number of factors, such as eating habits, disorders of the intestinal flora (2) and the immune system (3). There is a lack of specific therapeutic target in UC; therefore, the strategy of UC treatment involves immune regulation and inhibition of inflammation $(4,5)$. There have been previous precedents of successful prevention and cure of inflammatory diseases using traditional Chinese herbal medicines $(6,7)$. Ding's herbal enema (DHEP) was created by Ding Zemin, an eighth-generation descendant of the Ding family. DHEP has been used in The Third Affiliated Hospital of Nanjing University of Chinese Medicine (Nanjing, China) for $>50$ years and has an excellent curative effect in IBD treatment (8). DHEP contains Lonicerae japonicae flos (Jinyinhua), Sanguisorba officinalis L. (Diyu), Bletilla striata (Thunb.) Rchb.F. (Baiji), Phellodendron chinense Schneid. (Huangbo), Coptis chinensis Franch (Huanglian) and Portulaca oleracea L. (Machixian). All of the constituents of the DHEP solution that are from traditional Chinese medicine.

Studies have demonstrated that Jinyinhua, Diyu and Baiji contain $\beta$-sitosterol $(9,10)$. $\beta$-sitosterol has immunomodulatory and anti-inflammatory activity, and is present in numerous plants (11). A number of studies have revealed that $\beta$-sitosterol can inhibit inflammation through the NF- $\kappa \mathrm{B}$ pathway (12-16). Animal experiments suggest that $\beta$-sitosterol can significantly reduce colonic shortening, disease activity index (DAI) and fetal hemoglobin content in mice (17). Additionally, $\beta$-sitosterol specifically increases the activity of $\mathrm{T}$ helper (Th) cells, and increases the activity of $\mathrm{T}$ cells and natural killer cells (18). 
The present study investigated the mechanism of action of DHEP and its possible active ingredient, $\beta$-sitosterol, in the treatment of UC. C57BL/6J mice were treated with $3.5 \%(\mathrm{w} / \mathrm{v})$ dextran sulfate sodium (DSS) for 7 days to generate an animal model of colitis. DHEP and $\beta$-sitosterol were administered for 7 days, and the results indicated that DHEP and $\beta$-sitosterol inhibited the inflammatory response and restored the Th17/regulatory $\mathrm{T}$ cell (Treg) balance by regulating intestinal microbiota and short-chain fatty acids (SCFAs).

\section{Materials and methods}

Animals. A total of 50 adult female C57BL/6 mice (6-8 weeks old), weighing 20-24 g, were obtained from Yangzhou University Comparative Medical Center (Yangzhou, China). Mice were fed with free access to food and drinking water and housed in a temperature-controlled room $\left(22 \pm 4^{\circ} \mathrm{C}\right)$ under a 12 -h dark-light cycle with a relative humidity of $50 \%$. Animal welfare and experimental procedures were carried out in accordance with the guidelines and the associated ethical regulations of The Experimental Animal Ethics Committee of the Nanjing Hospital of Chinese Medicine Affiliated to Nanjing University of Chinese Medicine (Nanjing, China), which approved the animal experiments (approval no. 2020-10-002).

Reagents. DHEP was obtained from The Third Affiliated Hospital of Nanjing University of Chinese Medicine (Nanjing, China). $\beta$-sitosterol was purchased from Beijing Solarbio Science \& Technology Co., Ltd. (cat. no. IS0690). Mesalazine (5-ASA) was purchased from Ethypharm SAS (cat. no. 160306). DSS (36-50 kDa) was purchased from MP Biomedicals, LLC (cat. no. 160110). IL-6 (cat. no. 12912), TNF- $\alpha$ (cat. no. 3707) and p65 (cat. no. 6956) antibodies were obtained from Cell Signaling Technology, Inc. Cyclooxygenase (COX)-2 (cat. no. BM4419) antibodies were obtained from Wuhan Boster Biological Technology, Ltd. IL-17A (cat. no. m1037864) and IL-10 (cat. no. ml037873) enzyme-linked immunosorbent assay (ELISA) kits were purchased from Shanghai Enzyme-Linked Biotechnology Co., Ltd.

Determination of $\beta$-sitosterol in DHEP. DHEP powder (20 g) was extracted using methanol $(100 \mathrm{ml} \mathrm{x} \mathrm{3)}$ at room temperature. After removal of methanol under reduced pressure $(\sim-0.1 \mathrm{Mpa})$, the aqueous brownish syrup $(120 \mathrm{ml})$ was suspended in $\mathrm{H}_{2} \mathrm{O}(100 \mathrm{ml})$ and then partitioned with petroleum ether $(50 \mathrm{ml}$ x 3$)$ to obtain $500 \mathrm{mg}$ petroleum ether-soluble fraction. The fraction was further fractionated on a silica gel column eluted with petroleum ether-acetone (from 1:0 to 0:1) to obtain eleven fractions (1-10) according to thin-layer chromatography analysis. Fraction three $(65 \mathrm{mg})$ was loaded onto a silica gel column and eluted with methanol-CHCl3 (20:80, $30: 70$ and $40: 60 ; 50 \mathrm{ml}$ of each mixture) to yield four subfractions (fractions 3-1 to 3-4). Fraction 3-2 was analyzed by gas chromatography. The analytes $(1 \mu \mathrm{l})$ were separated using an Agilent 6890N-5975B GC system (Agilent Technologies, Inc.) equipped with a flame ionization detector and an HP-5 column (30 m x $250 \mu \mathrm{m} \times 0.25 \mu \mathrm{m}$ ) using $99.99 \%$ nitrogen as a carrier gas and $99.99 \%$ hydrogen as an auxiliary gas at a flow rate of $1.2 \mathrm{ml} / \mathrm{min}$ with a split ratio of $10: 1$. The temperatures of injector and detector were $250^{\circ} \mathrm{C}$ and $280^{\circ} \mathrm{C}$, respectively.
The oven temperature was gradually increased from $100^{\circ} \mathrm{C}$ to $250^{\circ} \mathrm{C}$ at a rate of $50^{\circ} \mathrm{C} / \mathrm{min}$ and from $250^{\circ} \mathrm{C}$ to $280^{\circ} \mathrm{C}$ at a rate of $10^{\circ} \mathrm{C} / \mathrm{min}$ and was held for $4 \mathrm{~min}$.

Animal models of DSS-induced acute colitis and treatments. Mice were treated with $3.5 \%$ (w/v) DSS in their drinking water for 7 days, followed by switching to regular drinking water for 7 days. Test compounds were administered via an enema per day for 7 days following DSS treatment. To investigate the effect of DHEP, all mice were randomly divided into 5 groups: Control (no DSS treatment, orally administered with sterile water; $n=12$ ), vehicle (DSS treated for 7 days, orally administered with sterile water; $n=12$ ), DHEP (administered by enema at a dose of $7.2 \mathrm{~g} / \mathrm{kg} ; \mathrm{n}=12$ ), 5-ASA (orally administered at a dose of $0.2 \mathrm{~g} / \mathrm{kg} ; \mathrm{N}=12$ ) and $\beta$-sitosterol (administered by enema at dose of $0.1 \mathrm{~g} / \mathrm{kg} ; \mathrm{n}=12$ ). At $24 \mathrm{~h}$ after the last enema, mice that had been fasting for $12 \mathrm{~h}$ were sacrificed.

Evaluation of DAI. Body weight, stool consistency and rectal bleeding of the mice were recorded daily and scored according to Cooper's scoring criteria (19). The mean of the three scores was calculated and recorded as the DAI. The body weight loss was scored as 0 when there was no loss in weight; 1 for a loss of $1-5 \% ; 2$ for a loss of $5-10 \%$; 3 for a loss of $10-15 \%$; or 4 for a loss of $>15 \%$. Regarding stool consistency, 0 points indicated normal pellets, 2 points indicated loose stools that did not stick to the anus and 4 points indicated diarrhea. For rectal bleeding, 0 points were given for negative (-) results in the Hemoccult test (20); 1 point for positive $(+)$ results; 2 points for positive $(++)$ results; 3 points for positive $(+++)$ results; and 4 points for gross rectal bleeding $(++++)$ results.

Histopathology. A representative sample from the middle region of the colon was fixed in $4 \%$ paraformaldehyde at $4{ }^{\circ} \mathrm{C}$ for $24 \mathrm{~h}$, embedded in paraffin, sectioned $(5-\mu \mathrm{m})$, stained with hematoxylin (at room temperature for $5 \mathrm{~min}$ ) and eosin (at room temperature for $2 \mathrm{~min}$ ) and then observed using an IX 51 epifluorescence Olympus microscope (Olympus Corporation) equipped with a DP-26 digital camera at x40 magnification.

16S DNA high-throughput sequencing. The construction of a high-throughput sequencing library and sequencing based on the Illumina MiSeq platform was performed by Genewiz, Inc. A Qubit 2.0 Fluorometer (Invitrogen; Thermo Fisher Scientific, Inc.) was used to detect the DNA concentration of the samples and to construct a sequencing library using the MetaVxTM Library Construction kit (Genewiz, Inc.). Using $10 \mathrm{nmol}$ of DNA as a template, a series of PCR primers designed by Genewiz, Inc. were used to amplify the prokaryotic $16 \mathrm{~S}$ ribosomal (r) DNA, including two highly variable regions of V3 and V4. The $\mathrm{V} 3$ and V4 regions were amplified using an upstream primer containing the 5'-CCTACGGRRBGCASCAGKVRVGAAT-3' sequence and a downstream primer containing the 5'-GGA CTACNVGGGTWTCTAATCC-3' sequence. In addition, a primer with an index was added to the end of the PCR product of the $16 \mathrm{~S}$ rDNA by PCR for next-generation sequencing. The library quality was determined using an Agilent 2100 Bioanalyzer (Agilent Technologies, Inc.) and library concentrations were determined using a Qubit 2.0 fluorometer (Invitrogen; Thermo Fisher Scientific, Inc.). After the DNA 
library was mixed, the Illumina MiSeq (Illumina, Inc.) instrument was used in accordance with the manufacturer's instructions. The instrument performed 2x300 bp double-end sequencing (paired end) reactions, and the sequence information was read by the MiSeq Control Software (v4.0) that comes with MiSeq.

SCFAs measurements. Fresh feces $(0.2 \mathrm{~g})$ were suspended in $1 \mathrm{ml}$ of deionized water and homogenized by vortexing for $1 \mathrm{~min}$ before centrifugation $\left(138,00 \mathrm{x} \mathrm{g}\right.$ at $4^{\circ} \mathrm{C}$ for $\left.30 \mathrm{~min}\right)$. The supernatant from the colon and cecum (630 and $720 \mu 1$, respectively) was mixed with $25 \%$ metaphosphoric acid at a ratio of 9:1 (v:v) and was shaken at $37^{\circ} \mathrm{C}$ for $4 \mathrm{~h}$. The supernatant was collected and stored at $-20^{\circ} \mathrm{C}$ before gas chromatography analysis. Standard curves were constructed according to a previously described method (15). Specifically, $1 \mu \mathrm{l}$ of the analytes was separated using an Agilent 6890N-5975B GC system equipped with an flame ionization detector and a DB-FFAP column (30 m x $250 \mu \mathrm{m} \times 0.25 \mu \mathrm{m}$; Agilent Technologies Inc.) (21) using $99.99 \%$ nitrogen as a carrier gas and $99.99 \%$ hydrogen as an auxiliary gas at a flow rate of $0.8 \mathrm{ml} / \mathrm{min}$ with a split ratio of 50:1. The temperatures of injector and detector were $250^{\circ} \mathrm{C}$ and $280^{\circ} \mathrm{C}$, respectively. The oven temperature was gradually increased from $60^{\circ} \mathrm{C}$ to $220^{\circ} \mathrm{C}$ at a rate of $20^{\circ} \mathrm{C} / \mathrm{min}$ and held for $1 \mathrm{~min}(22)$.

Flow cytometry. The Treg and Th17 cells in the mouse spleen and mesenteric lymph nodes (MLNs) were isolated using a Mouse Lymphocyte Separation Solution kit (cat. no. DKW33-R0100; Dakewe Biotech Co., Ltd.) according to the manufacturer's instructions. The differentiation of Treg and Th17 cells in the spleen and MLNs were analyzed with extracellular staining, membrane permeabilization, fixation, intracellular staining were performed using BD Pharmingen ${ }^{\mathrm{TM}}$ Transcription Factor Buffer Set kit (cat. no. 562574; BD Biosciences) according to the manufacturer's instructions, and flow analysis was conducted. To analyse the Treg cells, lymphocyte cells were stained with FITC-conjugated anti-CD4 (1:500; cat. no. 557307; BD Pharmingen; BD Biosciences) and P-phycoerythrin (PE)-conjugated anti-Foxp3 (1:500; cat. no. 12-4771-82; eBioscience; Thermo Fisher Scientific, Inc.). For Th17 cells, lymphocyte cells were stained with allophycocyanin-conjugated anti-CD4 (1:400; cat. no. 100411; BioLegend, Inc.) and PE-conjugated anti-IL-17A (1:500; cat. no. 506903; BioLegend, Inc.). The samples were analysed by BD Accuri C6 flow cytometry (BD Biosciences), and the data was analysed by FlowJo v10 software (FlowJo LLC).

Enzyme-linked immunosorbent assay (ELISA). The analyses of the fecal calprotectin (cat. no. m1037105, Shanghai Enzyme-Linked Biotechnology Co., Ltd.) (23), IL-10 (from colon tissues) and IL-17A (from colon tissues) were performed using the corresponding ELISA kit. The experimental procedure was based on the manufacturer's instructions. The fluorescence was measured using a microplate reader (BioTek Instruments, Inc.) with excitation at $450 \mathrm{~nm}$.

Determination of myeloperoxidase (MPO) activity. The MPO activity in colon tissues and serum was determined using an MPO detection kit (cat no. A044-1-1; Nanjing Jiancheng
Bioengineering Institute) according to the to the manufacturer's protocols.

Western blotting. Mouse colon tissue was collected and lysed in RIPA lysis buffer (cat. no. P0013B; Beyotime Institute of Biotechnology). The protein content was determined by the BCA method. Equal quantities $(50 \mu \mathrm{g})$ of protein lysate were loaded into each lane of a $10 \%$ SDS-polyacrylamide gel and wet-transferred onto a polyvinylidene difluoride membrane (Bio-Rad Laboratories, Inc.) using a Bio-Rad electro transfer apparatus. After blocking with 5\% skimmed milk powder in Tris-buffered saline and $0.1 \%$ Tween-20 at room temperature for $2 \mathrm{~h}$, the membranes were incubated with the primary antibodies. The following antibodies were used: p65 (1:500), COX-2 (1:500), IL-6 (1:1,000), TNF- $\alpha$ (1:100) and actin (cat. no. ab6276, 1:5,000; Epitomics; Abcam). Horseradish peroxidase (HRP)-conjugated goat anti-rabbit IgG antibody (cat. no. D110058, 1:10,000; Sangon Biotech Co., Ltd.) was used as the secondary antibody. Immunoblotted bands were visualized with the Immobilon Western Chemilum HRP Substrate (cat. no. WBKLS0100; MilliporeSigma) using a JS-680 B Gel Documentation and Analysis System (Bio-Rad Laboratories, Inc.) and quantified using Image Lab v3.0 (Bio-Rad Laboratories, Inc.) and Quantity One v4.62 (Bio-Rad Laboratories, Inc.) software. All the proteins were normalized to the corresponding actin level.

Statistical analysis. Statistical analysis was performed using one-way ANOVA, followed by Tukey's post hoc test. Ordinal data were analyzed using Kruskal-Wallis with Dunn's post hoc test. Quantitative data are presented as mean \pm standard deviation (unless otherwise shown). $\mathrm{P}<0.05$ was considered to indicate a statistically significant difference. The data presented represent at least three independent experiments. The 16S rDNA data was analysed by QIIME data analysis package (v1.9.1; http://qiime.org/); the $\alpha$ diversity was represented by the Shannon indexes, and the $\beta$ diversity represented by principal coordinate analysis (PCA). Sequences were grouped into operational taxonomic units (OTUs) using the clustering program VSEARCH (v1.9.6; https://github.com/torognes/vsearch) against the Silva 119 database (https://www.arb-silva.de/) pre-clustered at $97 \%$ sequence identity. Linear discriminant analysis effect size (LEfSe; http://huttenhower.sph.harvard. edu/galaxy/) to obtain the OTU for each group. Differences in relative abundances of OTUs were calculated using Tukey's honest significant difference test. Data were analysed by the GraphPad Prism 7.0 software (GraphPad Software, Inc.).

\section{Results}

HEP and $\beta$-sitosterol attenuates DSS-induced UC in mice. C57BL/6 mice were treated with $3.5 \%(\mathrm{w} / \mathrm{v}) \mathrm{DSS}$ for 7 days to induce UC (Fig. 1A) (24). On day 7, the bleeding score (Fig. 1C), diarrhea score (Fig. 1D), DAI (Fig. 1E) and calprotectin content (an indicator of colitis (Fig. 1G) (24-26) were increased in mice treated with DSS compared with the vehicle, whereas the body weight (Fig. 1B) was decreased. In addition, compared with the control group, the mice body weight was significantly shortened when DSS treated with DSS for 7 days (Fig. 1F). These results indicated that the mouse 
A

C

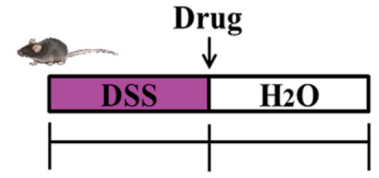

$\begin{array}{llllllllll}1 & 2 & 3 & 4 & 5 & 6 & 7 & 8 & 9 & 1011121314\end{array}$

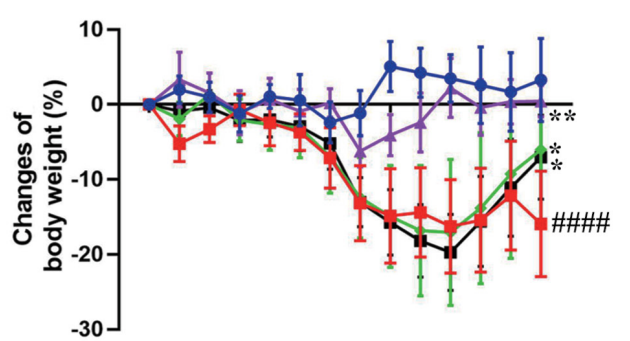

Days

D

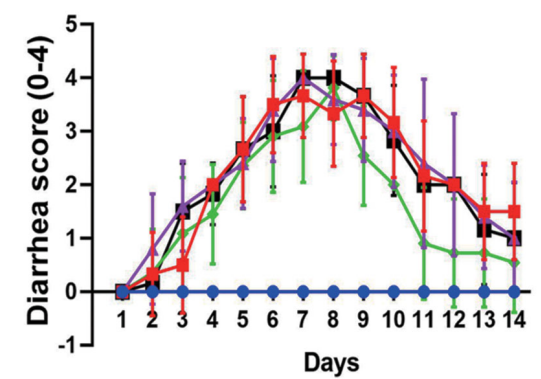

$\mathbf{F}$

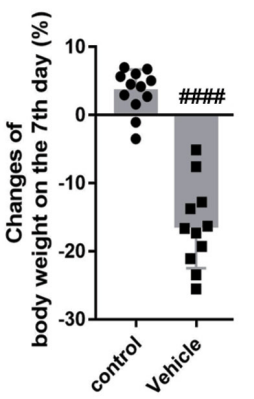

G

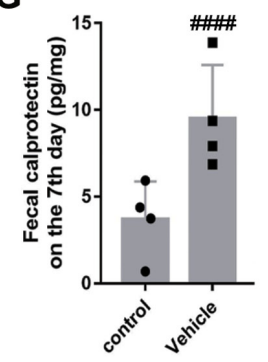

I

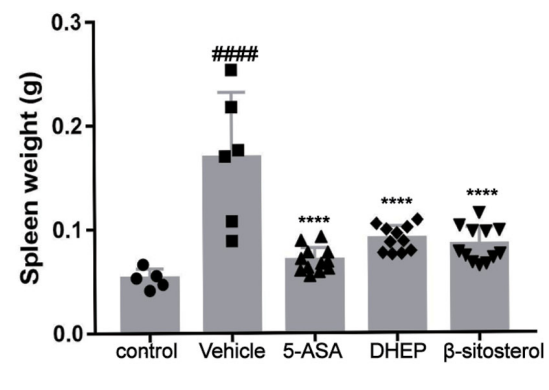

K $\rightarrow$ control

$\rightarrow-$ Vehicle

\pm 5-ASA

$\rightarrow$ DHEP

B

$+\beta$-sitosterol

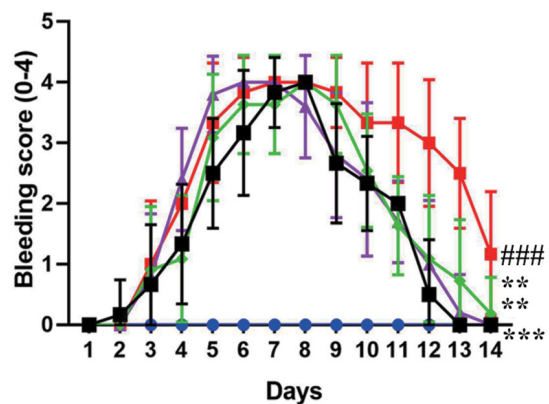

E
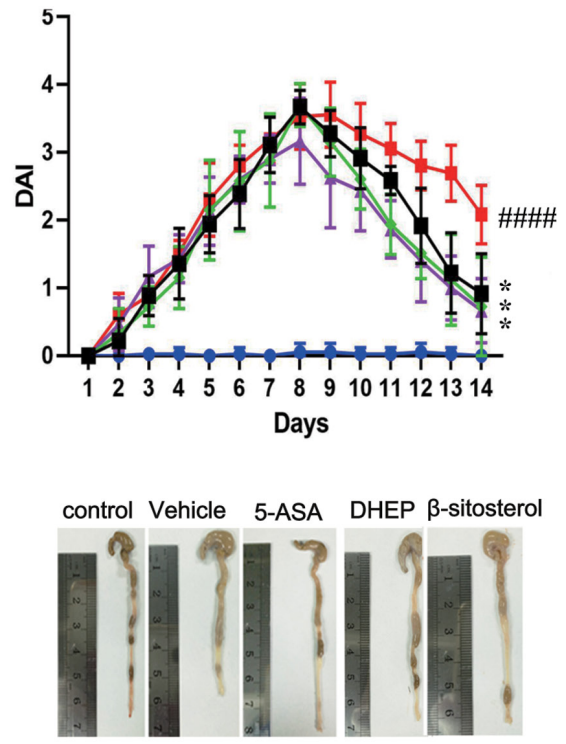

J

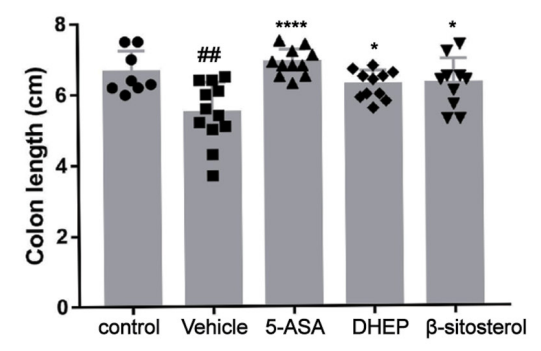

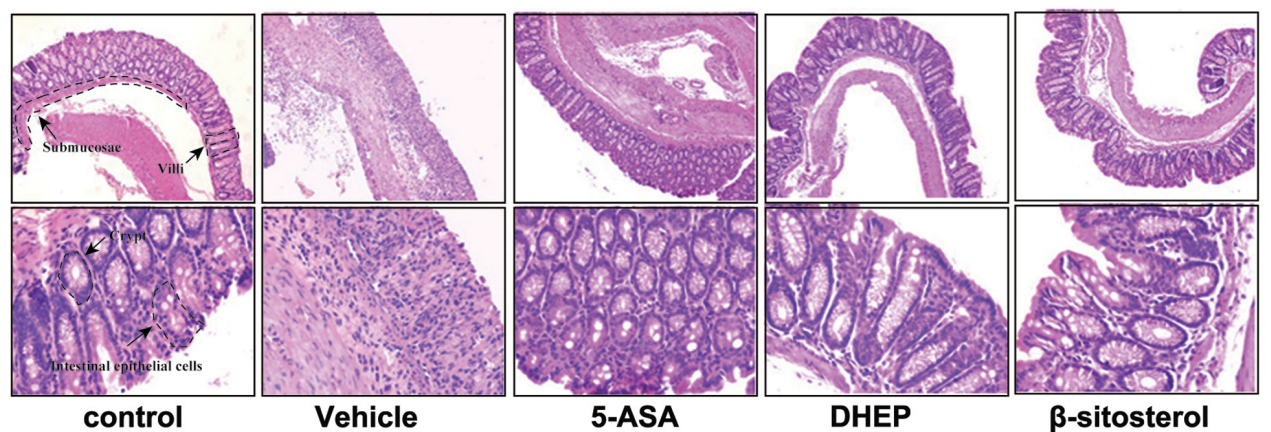

Figure 1. DHEP and $\beta$-sitosterol treatment of acute colitis in vivo. (A) Schematic overview of the experimental design. Changes in (B) body weights, (C) clinical bleeding scores, (D) clinical diarrhea scores and (E) DAI are presented, and all statistical values are compared on day 14. (F) Weight loss and (G) fecal calprotectin content in feces after colitis induction. $(\mathrm{H})$ Representative images of whole colons and colon length. (I) Spleen mass of mice after the end of the experiment. (J) Quantification of whole colons and colon length. (K) Colon sections were counterstained with hematoxylin and eosin, and high-magnification images are presented in the bottom row (top row magnification, $\mathrm{x} 100$; bottom row, $\mathrm{x} 400$ ). $\mathrm{n}=4-12 .{ }^{\# \#} \mathrm{P}<0.01,{ }^{\# \# \#} \mathrm{P}<0.005$ and ${ }^{\# \# \# "} \mathrm{P}<0.001$ vs. control group; ${ }^{*} \mathrm{P}<0.05,{ }^{* *} \mathrm{P}<0.01$, ${ }^{* * *} \mathrm{P}<0.005$ and ${ }^{* * * *} \mathrm{P}<0.001$ vs. vehicle group. DHEP, Ding's herbal enema; DAI, disease activity index; 5-ASA, mesalazine; DSS, dextran sulfate sodium. 
model was successfully established $(27,28)$. DHEP, compared with the vehicle group, was administered by enema for 7 days and attenuated the symptoms of colitis (loss of body weight, diarrhea, rectal bleeding and DAI) (Fig. 1B-E). In addition, for $\beta$-sitosterol treatment, the loss of body weight, rectal bleeding and DAI score were relieved remarkably compared with the vehicle group (Fig. 1B-E). These results suggested that DHEP and $\beta$-sitosterol might be a positive effect on the treatment of DSS-induced UC mice model.

As presented in Fig. $1 \mathrm{H}$ and $\mathrm{J}$, the colon was edematous and significantly shortened in the vehicle group of mice compared with that in the control group. A reduction in colon length in DSS-treated mice was significantly attenuated by treatment with 5-ASA, DHEP and $\beta$-sitosterol. Additionally, 5-ASA, DHEP and $\beta$-sitosterol significantly reduced the weight of the spleen compared with the vehicle, which was the largest immune organ (Fig. 1I). Comparison with the control group mice identified certain pathological changes in the DSS-treated mice (Fig. 1K), including damaged intestinal epithelial cells, submucosal edema, disappearance of villi and crypts and a large number of infiltrating inflammatory cells (Fig. 1K). However, these pathological changes were relieved after administration of 5-ASA, DHEP and $\beta$-sitosterol (Fig. 1K).

DHEP and $\beta$-sitosterol remodels the distribution of intestinal microbiota in mice. To determine whether intestinal microbiota were affected by treatment with 5-ASA, DHEP and $\beta$-sitosterol, 16S rDNA sequencing was used to evaluate the abundance of intestinal flora in mice. After the sequencing data were analyzed by QIIME, the $\alpha$ diversity for all groups represented by the Shannon indexes is presented in Fig. 2A. Notably, the diversity in the DSS-treated groups were similar to that in the control group. By contrast, the diversity in the 5-ASA group was significantly decreased compared with that in the vehicle group. However, these differences were not significant in the DHEP- and $\beta$-sitosterol-treated groups (Fig. 2A). $\beta$ diversity represented by PCA is presented in Fig. $2 \mathrm{~B}$. The distributions in the DHEP and 5-ASA groups were relatively concentrated, and the distribution between the groups was relatively dispersed. There was no overlap between the DHEP and 5-ASA groups. There was a partial overlap between the vehicle and $\beta$-sitosterol groups and the control group; however, the overall distribution was relatively dispersed, which may be due to individual differences and intervention effects in mice. The bacterial composition at the phylum level is presented in Fig. 2C. The abundance at this level was variable, including Firmicutes, Bacteroidetes and Proteobacteria. The abundance of Bacteroidetes and Proteobacteria were increased in DHEP group compared with vehicle group, but the abundance of Firmicutes was decreased. These results indicated that DHEP might be able to regulate the ratios of Bacteroidetes/Firmicutes and Proteobacteria/Firmicutes. In addition, in the $\beta$-sitosterol group, the ratios of Bacteroidetes/ Firmicutes and Proteobacteria/ Firmicutes were upregulated, but not to the same level as the DHEP group.

To identify the bacteria possibly regulated by DHEP and $\beta$-sitosterol, LEfSe analysis was used to obtain the OTUs for each group. As a result, 42 significant OTUs were identified, along with their linear discriminant analysis score (Fig. 2D), and a heatmap was generated (Fig. 2E). Notably, 39 OTUs were significantly altered in the control, 5-ASA, DHEP and $\beta$-sitosterol groups (Fig. $2 \mathrm{~F}$ ). Comparison with the vehicle group indicated that 5-ASA altered 21 OTUs. The 12 reduced OTUs in the 5-ASA group (OTU 42, 321, 8, 7, 43, 182, $100,203,183,48,125$ and 286) are highlighted in red, and 9 increased OTUs (OTU 73, 16, 13, 60, 77, 39, 41, 136 and 71) are highlighted in green in Fig. 2F. In the DHEP-treated group, 19 OTUs were altered compared with those in the vehicle group. As presented in Fig. 2F, 11 OTUs (OTU 321, $12,120,182,239,203,83,233,183,93$ and 196) were reduced and are highlighted in red, which belonged to the genera of Erysipelatoclostridium, Bifidobacterium, Anaerotruncus, Defluvitaleaceae UCG-011, Family XIII AD3011 group, Tyzzerella, Eubacterium coprostanoligenes, Proteus and three uncultured genera; and a total of seven OTUs (OTU 73, 3, 13, 29, 41, 136 and 1) were increased, which belonged to the genera of Bacteroides, Lactobacillus, Turicibacter, Parabacteroides, Eubacterium xylanophilum group and one uncultured genus. In the $\beta$-sitosterol-treated group, six OTUs (OTU 22, 182, 100, 203, 93 and 286) were reduced (Fig. 2F). The six reduced OTU belonged to Akkermansia, Defluviitaleaceae UCG-011, Ruminococcaceae UCG-005, Tyzzerella and Oligella Only four OTUs were increased (OTU 270, 13, 29 and 41), which belonged to Lactobacillus, Parabacteroides and two uncultured genera. Thus, 34 OTUs were altered by 5-ASA, DHEP and $\beta$-sitosterol, and only four OTUs (OTU 182, 203, 13 and 41) were modulated in the same direction as in the control group for all three treatment groups. These four OTUs belonged to the genera Anaerotruncus, Ruminococcaceae UCG-005 and Turicibacter and uncultured bacterium, which belonged to the Firmicutes, Firmicutes, Bacteroidetes and Firmicutes phyla, respectively (Fig. 2G).

DHEP and $\beta$-sitosterol treatment increases the profiles of SCFAs in the colon of mice with DSS-induced UC. SCFAs are the end-products generated from food with a high dietary content by gut microbiota and can alleviate IBD in animal models (29). To evaluate whether DHEP and $\beta$-sitosterol increased the release of SCFAs, the SCFA content in the colon of mice was evaluated (Fig. 3). The levels of SCFAs, including acetic acid (Fig. 3A and B), propionic acid (Fig. 3C and D) and butyric acid (Fig. 3E and F) were significantly reduced in the colon of DSS-treated mice compared with the control group, and the levels of SCFAs were increased in the 5-ASA, DHEP and $\beta$-sitosterol groups compared with the vehicle group. These results indicated that the beneficial effects of DHEP in colitis involved an increase in the SCFA content in the colon.

DHEP and $\beta$-sitosterol regulates the Th17/Treg balance in mice with DSS-induced UC. The Th17/Treg transformation balance is important for the maintenance of intestinal homeostasis (30). To investigate whether DHEP and $\beta$-sitosterol had a positive effect on the balance of Th17/Treg cells, the expression levels of IL-17A and IL-10, which are specifically expressed in Th17 cells and Treg cells, respectively, were determined. The results demonstrated that the numbers of Treg cells in the MLNs (Fig. 4A) and spleen (Fig. 4B) of mice with DSS-induced UC were significantly lower compared with those in the control mice. After administration with 5-ASA or $\beta$-sitosterol for 7 days, the number of Treg cells was significantly increased 
A

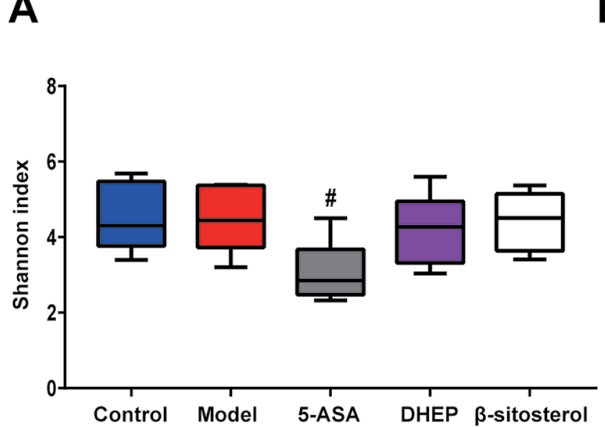

B

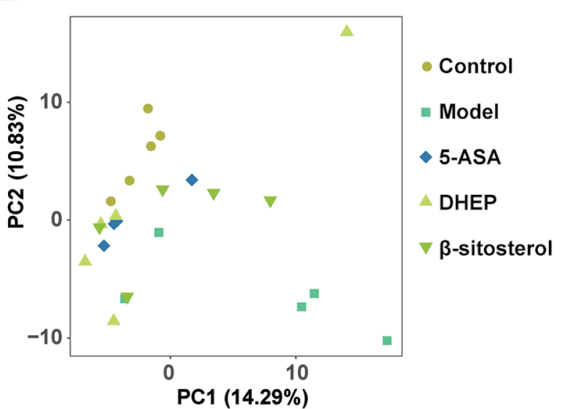

C 1.00

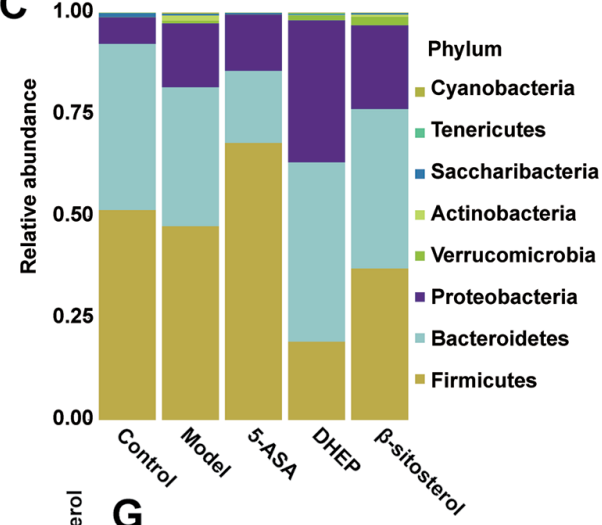

$\mathbf{G}$

D

E

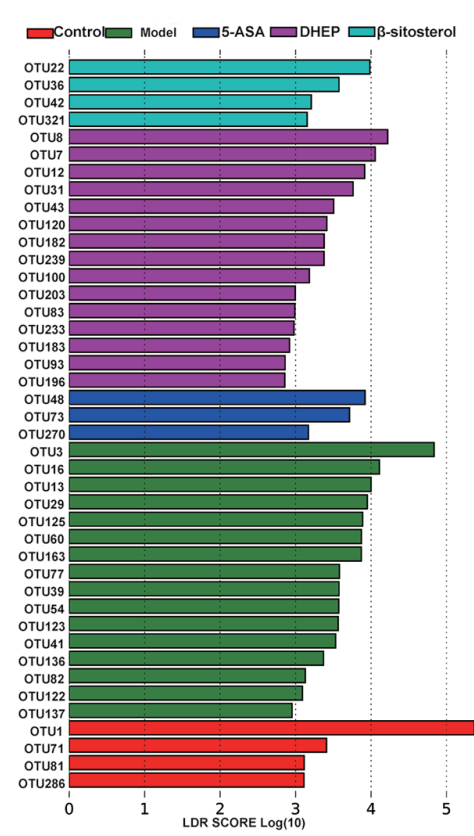

$E$

Model 5-ASA

$F_{-}$
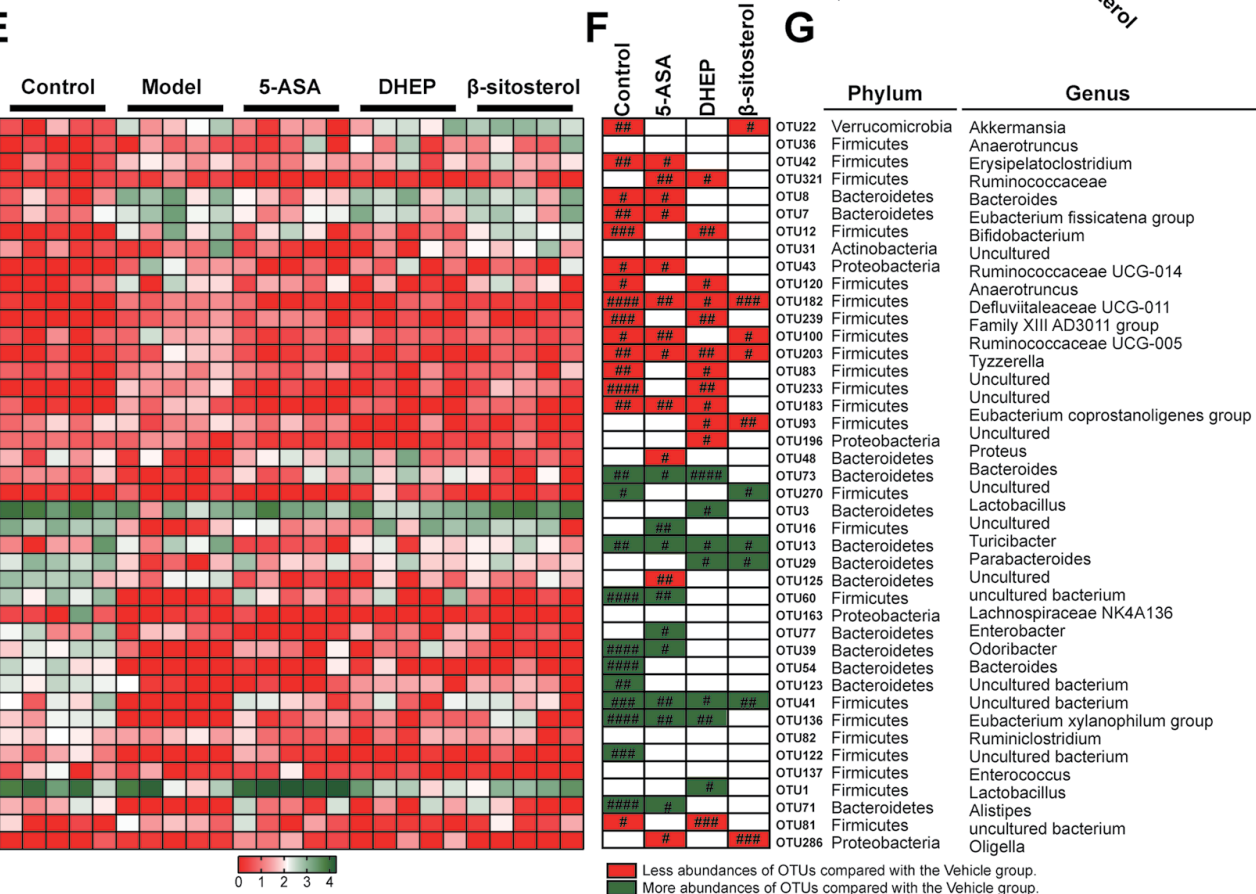

Less abundances of OTUs compared with the Venicle group.
More abundances of OTUs compared with the Vehicle group.

Figure 2. DHEP and $\beta$-sitosterol treatments affects the distribution of flora in vivo. (A) Comparison of $\alpha$ diversity assessed by Shannon index-based genus level (B) PC analysis of gut microbiota communities-based genus level. (C) Column diagram of microbial composition at the phylum level. (D) Linear discriminant analysis at the OTU level. (E) Heatmap showing the abundance of 42 OTUs in different groups based on linear discriminant analysis. (F) Changing direction of OTUs induced by DSS, 5-ASA, DHEP or $\beta$-sitosterol intervention. $(\mathrm{G})$ OTUs represent bacterial taxonomic information ( $\mathrm{n}=5$ ). ${ }^{\sharp} \mathrm{P}<0.05$ vs. vehicle group. DHEP, Ding's herbal enema; OTU, operational taxonomic unit; DSS, dextran sulfate sodium; 5-ASA, mesalazine; PC, principal coordinate.

in MLNs (Fig. 4A) but markedly decreased in spleen (Fig. 4B) compared with the vehicle. In the DHEP-treated mice group, the number of Treg cells was significantly increased in both the MLNs (Fig. 4A) and spleen (Fig. 4B) compared with the vehicle. As presented in Fig. 4C, treatment with 5-ASA and $\beta$-sitosterol significantly decreased the number of Th17 cells in the spleen compared with that in the vehicle group; however, DHEP did not demonstrate this effect. Unexpectedly, the IL-17A level was significantly decreased in the control group compared with the vehicle (Fig. 4E). Additionally, 5-ASA, DHEP and $\beta$-sitosterol-treated mice manifested an opposite trend in their IL-17A and IL-10 expression levels. IL-10 was significantly increased compared with the vehicle (Fig. 4D), and the IL-17A expression level was significantly reduced compared with the vehicle (Fig. 4E).

DHEP and $\beta$-sitosterol reduces inflammation in the DSS-induced colitis. Fecal calprotectin is an acute inflammatory marker that was significantly increased in the
DSS-treated vehicle group of mice compared with the control; in addition, it was significantly reduced in 5-ASA-, DHEPand $\beta$-sitosterol-treated mice (Fig. 5A). High levels of MPO enzyme are expressed in neutrophils, reflect the infiltration of neutrophils and indirectly correspond to inflammation in certain tissues (31). MPO levels in the tissue and serum were significantly upregulated in the vehicle group of mice compared with the control, and significantly downregulated in the 5-ASA-, DHEP- and $\beta$-sitosterol-treated mice compared with the vehicle (Fig. 5B and C). p65 (Fig. 5D), IL-6 (Fig. 5F) and TNF- $\alpha$ (Fig. 5G) were secreted in the colon of DSS-treated mice; the expression levels of p65 and IL-6 were significantly increased in the vehicle group compared with the control, and significantly reduced by treatment with 5-ASA and $\beta$-sitosterol compared with the vehicle. TNF- $\alpha$ followed this same pattern; however, the changes in expression level were not significant. However, DHEP only lowered the secretion of p65 compared with the vehicle (Fig. 5D). 5-ASA and DHEP treatment also reduced the protein expression level of COX-2 in DSS-treated 

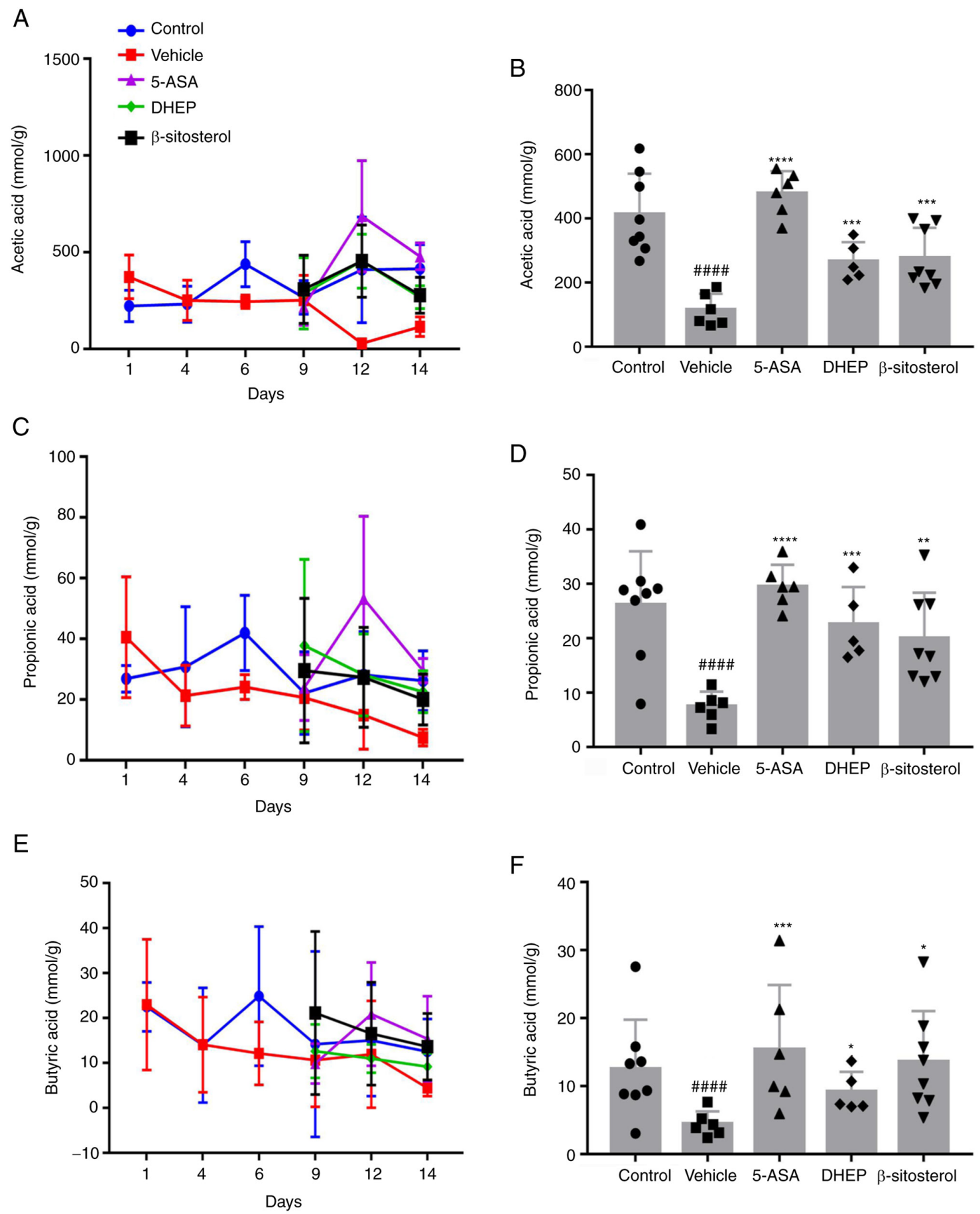

Figure 3. DHEP and $\beta$-sitosterol can increase short chain fatty acid content in vivo. (A) Acetic acid content across the 14-day period. (B) Acetic acid content at day 14 (C) Propionic acid content across the 14-day period. (D) Propionic acid content at day 14. (E) Butyric acid content across the 14-day period. (F) Butyric acid content at day 14. $\mathrm{n}=5-8 .{ }^{\# \# \# "} \mathrm{P}<0.001$ vs. control group; ${ }^{\mathrm{P}} \mathrm{P}<0.05,{ }^{* *} \mathrm{P}<0.01,{ }^{* * * *} \mathrm{P}<0.005$ and ${ }^{* * * * *} \mathrm{P}<0.001$ vs. vehicle group. DHEP, Ding's herbal enema; 5-ASA, mesalazine.

mice compared with the vehicle, which was detected in the pathological state of inflammation; $\beta$-sitosterol had no effect on the COX-2 level (Fig. 5E and H).

\section{Discussion}

DHEP is a traditional Chinese medicine used as an enema treatment, which was developed by Professor Xie Jinyu and Professor Ding Zemin (8). DHEP is effective in the clinical treatment of UC (8). The present study determined a preliminary mechanism of action of DHEP treatment in DSS-induced
UC and analyzed the content of $\beta$-sitosterol in DHEP by gas chromatography. The results presented in Fig. S1 indicated that the concentration of $\beta$-sitosterol in DHEP was $\sim 13.9 \mathrm{mg} / \mathrm{g}$. The DHEP dose in mice was determined according to the clinical dose. The clinical dose of DHEP in Nanjing Hospital of Chinese Medicine Affiliated to Nanjing University of Chinese Medicine is $0.58 \mathrm{~g} / \mathrm{kg} / \mathrm{day}$; according to the dose conversion between human and mice (mouse dose = human dose $x$ 12.33) (32), the theoretical dose for mouse administration was $7.15 \mathrm{~g} / \mathrm{kg} / \mathrm{day}$. Hence, a dose of $7.2 \mathrm{~g} / \mathrm{kg} /$ day was used in the present study. Thus, the concentration of $\beta$-sitosterol 
A

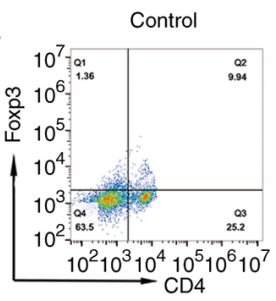

$\mathrm{B}$
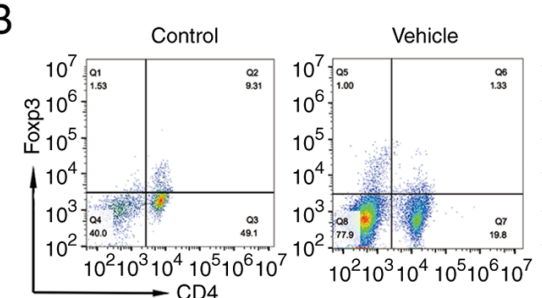

$10^{2} 10^{3} 10^{4} 10^{5} 10^{6} 10^{7}$
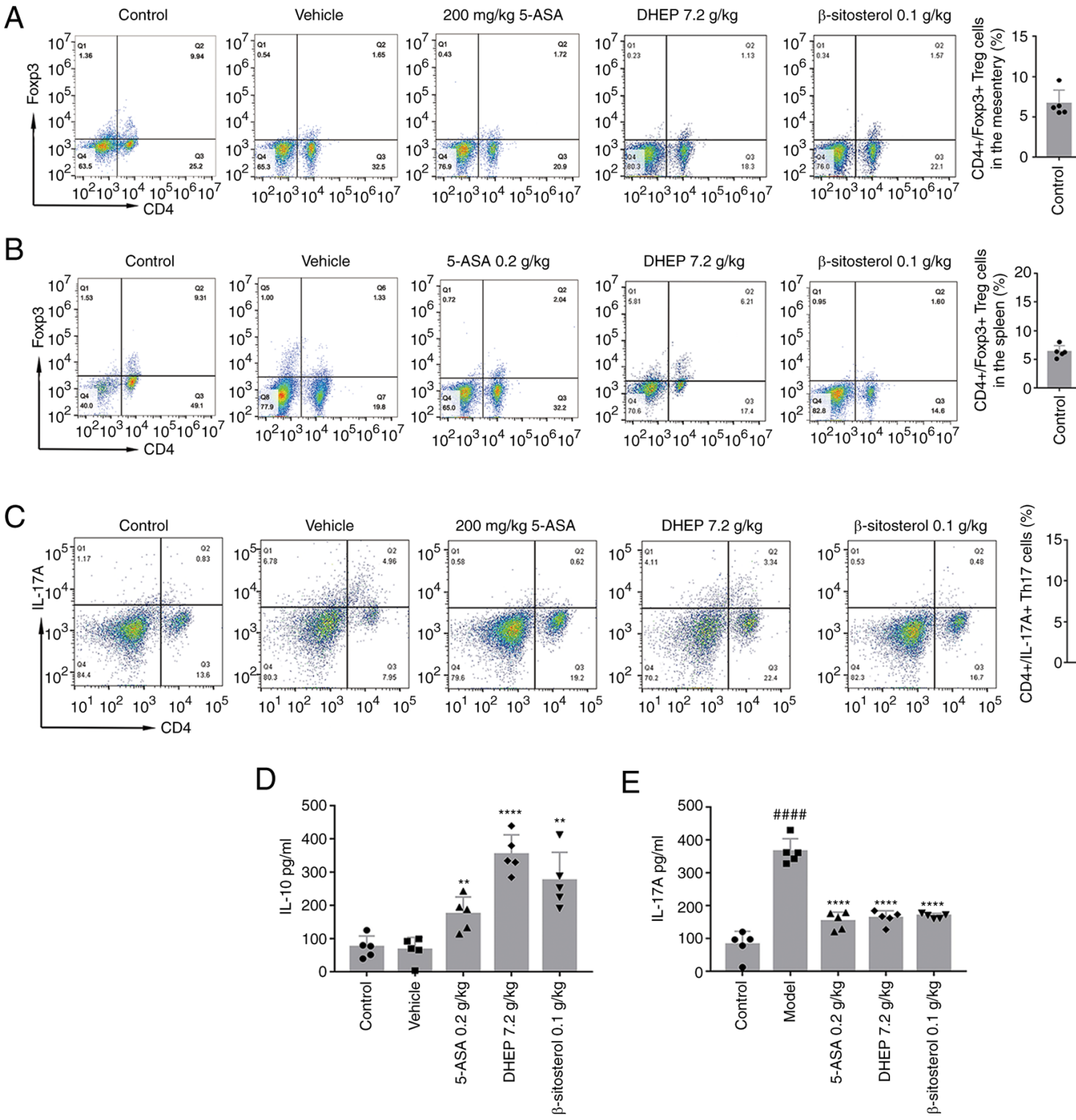

E

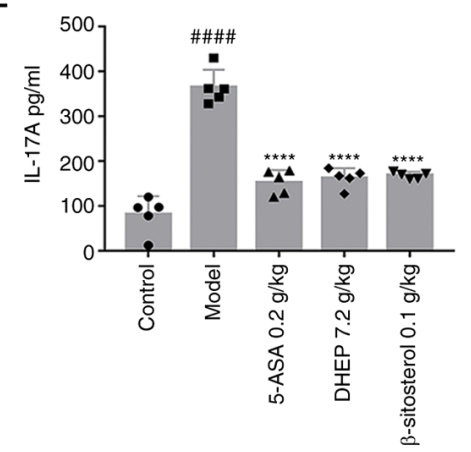

Figure 4. DHEP and $\beta$-sitosterol treatments promote $\mathrm{T}$ cell differentiation in mice. Representative flow cytometric profiles and quantitative analysis of the (A) spleen and (B) mesenteric lymph nodes Treg cells, and (C) spleen Th17 cells $(\mathrm{n}=3-9)$. ELISA showing (D) IL-10 and (E) IL-17A protein expression levels $(\mathrm{NN}=5)$. ${ }^{\# \#} \mathrm{P}<0.01,{ }^{\# \# *} \mathrm{P}<0.005$ and ${ }^{\# \# \# *} \mathrm{P}<0.001$ vs. control group; ${ }^{* *} \mathrm{P}<0.01,{ }^{* * * *} \mathrm{P}<0.005$ and ${ }^{* * * * *} \mathrm{P}<0.001$ vs. vehicle group. DHEP, Ding's herbal enema; 5-ASA, mesalazine; Th, T helper cell; Treg, regulatory T cell.

in DHEP was $\sim 13.9 \mathrm{mg} / \mathrm{g}$, and the dose of $100 \mathrm{mg} / \mathrm{kg} / \mathrm{day}$ of $\beta$-sitosterol was selected as the therapeutic dose.

Intestinal homeostasis depends on the dynamic interaction between intestinal flora, intestinal epithelial cells and immune cells (33). These microorganisms coexist with humans and play a notable role in the regulation of the mammalian immune system. The homeostasis of the ecosystem formed by the gut microbiota and the host is important for human health $(34,35)$. Intestinal flora plays a role in the pathogenesis of IBD. The present study analyzed the gut microbiota via next-generation sequencing. The results of LEfSe analysis at the OTU level indicated differences in the abundance between five groups. In the DSS-treated vehicle group, 15 OTUs were significantly increased and 11 OTUs were considerably decreased. Increased OTUs were assigned to the Akkermansia, Bacteroides and Anaerotruncus genera that were the most abundant and extensively studied members of the commensal microbiota. These bacteria benefit the host by fermentation of complex dietary carbohydrates and modulation of mucosal glycosylation (36). Additionally, genera with increased abundance included Erysipelatoclostridium, Ruminococcaceae, Ruminococcaceae UCG-014, Defluviitaleaceae UCG-011 and Ruminococcaceae UCG-005 of the Firmicutes phylum. The abundance of the Firmicutes phylum in the colons of patients with IBD was increased (36). Comparison with the vehicle group indicated that 11 OTUs were reduced and seven OTUs were increased. Reduced OTUs belonged to the Firmicutes phylum, except OTU 196, which belonged to the Proteobacteria phylum. Additionally, four out of the seven reduced OTUs (OTU 73, 3,13 and 29) belonged to the Bacteroidetes phylum and three OTUs (OTU 41, 136 and 1) belonged to the Firmicutes phylum. These results suggested that DHEP may rebalance the flora that was altered by DSS treatment. 
A
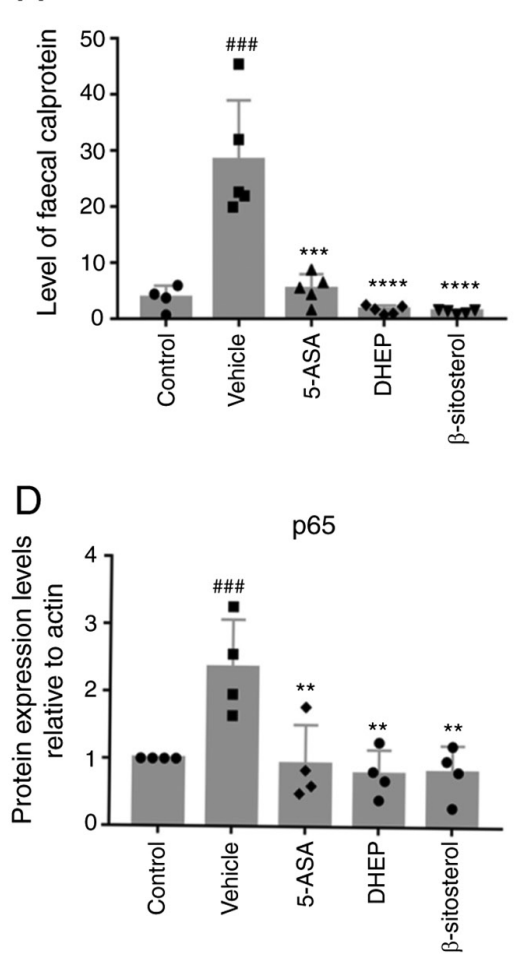

G

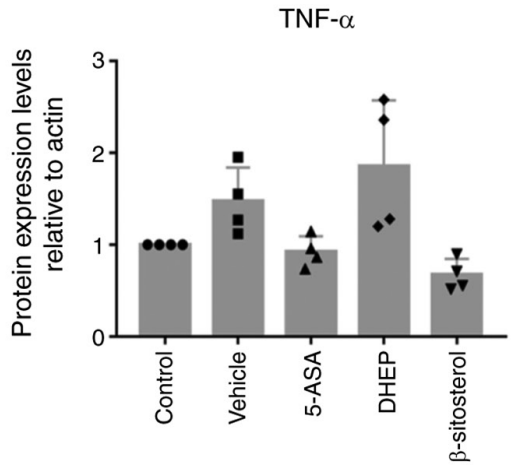

C
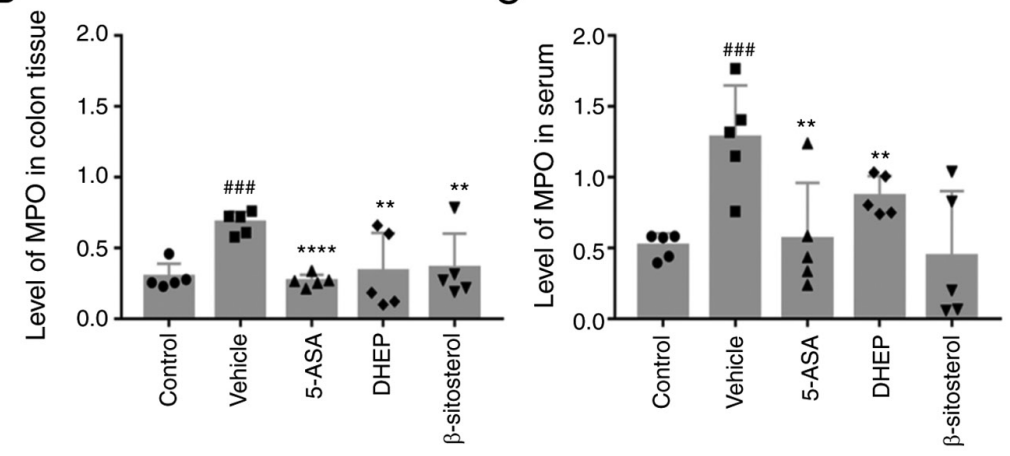

E

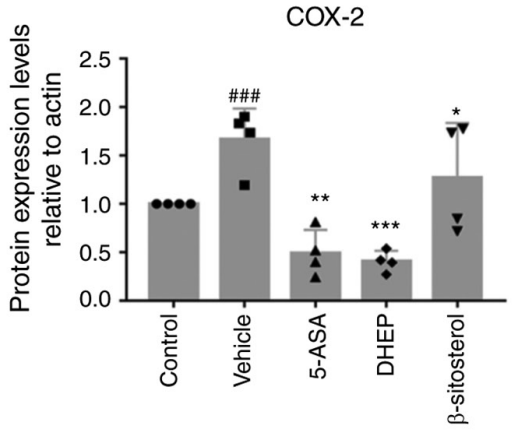

F

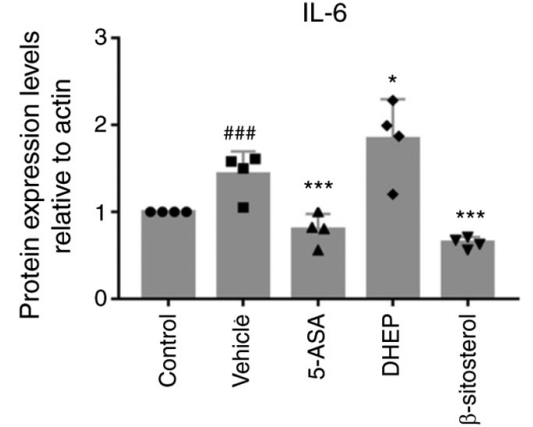

$\mathrm{H}$

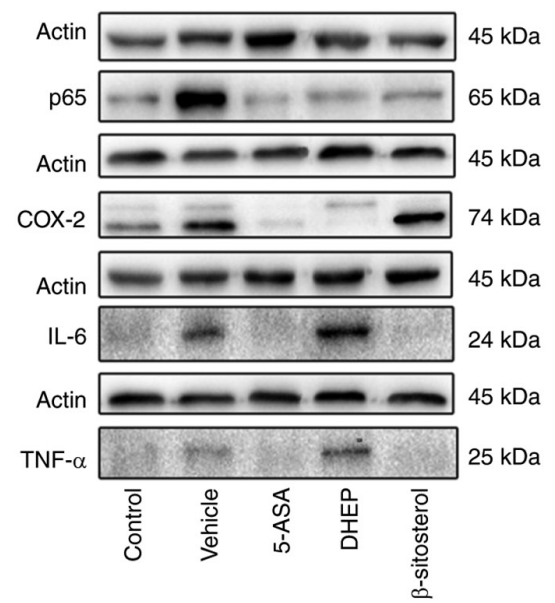

Figure 5. Expression levels of various inflammatory factors in mice. (A) Fecal calprotectin content in feces after sacrifice of the mice. MPO content in (B) colon tissue and (C) serum of mice. Quantification of western blotting showing (D) p65, (E) COX-2, (F) IL-6 and (G) TNF- $\alpha$. (H) Western blot analysis of actin, p65, COX-2, IL-6 and TNF- $\alpha$. N=4-6. ${ }^{\# \# \#} \mathrm{P}<0.001$ vs. control group; ${ }^{*} \mathrm{P}<0.05,{ }^{* *} \mathrm{P}<0.01,{ }^{* * *} \mathrm{P}<0.001$ and ${ }^{* * * *} \mathrm{P}<0.0001$ vs. vehicle group. MPO, myeloperoxidase; COX-2, cyclooxygenase-2; 5-ASA, mesalazine; DHEP, Ding's herbal enema.

SCFAs, such as acetate, n-propionate and n-butyrate, are derived by microbial fermentation of dietary fiber in the intestine (37). An increasing number of studies have revealed that SCFAs are critical for the regulation of the immune system, especially butyric acid (38), and that SCFAs can promote the development of Treg cells and inhibit the development of Th17 cells $(39,40)$. The balance between these two cell types is needed for immune regulation and can regulate the immune function of the body (41). Th17 cells are mainly present on the intestinal mucosal barrier surface to protect the host from microbial invasion through the epithelium (42). Treg cells are mainly present in the intestinal mucosa and control the excessive immune response caused by $\mathrm{T}$ cells that may damage the host tissue. Mature Th17 cells can express a variety of effectors molecules, including IL-17, TNF- $\alpha$, IL-17A and other cytokines $(42,43)$. IL-17A plays an important role in the recruitment, activation and migration of granulocytes (44). These processes accelerate the polarization of Th17 cells, leading to intestinal inflammation (45). The present study demonstrated that $\beta$-sitosterol and DHEP could significantly increase the SCFA content in the intestine of mice, particularly the amount of butyric acid. DHEP and $\beta$-sitosterol significantly increased the proportion of Tregs in the MLNs, and there were no significant differences in the ratio of Tregs between DHEP and $\beta$-sitosterol treatments.

$\beta$-sitosterol has immunomodulatory and anti-inflammatory activity, and is found in numerous plants (11). A large number of studies have indicated that $\beta$-sitosterol can inhibit inflammation through the NF- $\kappa \mathrm{B}$ pathway (12-16). However, the present study 
suggested that $\beta$-sitosterol can significantly reduce colonic shortening, DAI and fecal hemoglobin content in mice. In addition, $\beta$-sitosterol specifically increases the activity of Th cells and increases the activity of $\mathrm{T}$ cells (18). In the present study, $\beta$-sitosterol significantly reduced body weight loss, diarrhea score, bleeding score, colon index and spleen index. In addition, both DHEP and $\beta$-sitosterol significantly increased the proportion of Tregs in the MLNs, and there was no significant difference in the ratio of Tregs between DHEP and $\beta$-sitosterol treatments. $\beta$-sitosterol could significantly reduce Th17 cells in the spleen, but DHEP had no significant effect. These results suggested that $\beta$-sitosterol could be used as a potential drug for the treatment of UC.

Thus, the current study demonstrated that $\beta$-sitosterol and the clinically effective DHEP enema solution protected mice from DSS-induced colitis. The present study identified four therapeutic mechanisms of both treatments, including effective inhibition of inflammatory responses, and regulation of intestinal microbiota and SCFAs to restore the Th17/Treg balance. However, additional comprehensive studies may be focused on the following three points: i) Investigation of the notable role of the gut microbiota in the mechanism of action; ii) identification of other active substances in DHEP that are effective in the treatment of UC in addition to $\beta$-sitosterol; and iii) identification of DHEP-induced changes in other intestinal flora metabolites in addition to SFCAs, which are important for the treatment of UC. Furthermore, the efficacy of the active ingredient $\beta$-sitosterol is clinically validated, thus simplifying the convenient administration of the preparation.

\section{Acknowledgements}

Not applicable.

\section{Funding}

This work was supported by Nanjing Medical Science and Technique Development Foundation (grant no. QRX17090), Nanjing Famous Traditional Chinese Medicine Studio (grant no. ZSM-2017-NJ), Multi-Disciplinary Integrated Diagnosis and Treatment Platform for Inflammatory Bowel Disease (grant no. 00302010524), Natural Science Foundation of Jiangsu Province (grant no. SBK20180140), Young Talent Cultivation Program of the Key Subject of 'Chinese Medicine Anorectal Diseases' of the State Administration of Chinese Medicine (grant nos. GCPY201701 and GCPY201902) and The Third Chinese Medicine Experts' Academic Experience Succession Work Project of Jiangsu Provincial (grant no. 2019-SSPSC-DK).

\section{Availability of data and materials}

The datasets generated and/or analyzed during the current study are available in the BioProject repository (https://www. ncbi.nlm.nih.gov/bioproject/PRJNA753609).

\section{Authors' contributions}

YYT and YD acquired and analyzed the data, and drafted and revised the manuscript. XZ, SMZ and GJD performed the animal experiments. XY, DCX and PC obtained and analyzed the 16s rDNA sequencing data. JMZ, JZM and ML obtained and analyzed the SCFAs measurement data. SCH and YL performed the flow cytometry experiment and data analysis. YTZ and HX performed the western blotting experiment and data analysis. KD and YJD designed the study, provided final approval of the version to be published and agreed to be accountable for all aspects of the work in ensuring that questions related to the accuracy or integrity of any part of the work are appropriately investigated and resolved. YJD and KD confirm the authenticity of all the raw data. All authors have read and approved the final manuscript.

\section{Ethics approval and consent to participate}

The Experimental Animal Ethics Committee of the Nanjing Hospital of Chinese Medicine Affiliated to Nanjing University of Chinese Medicine approved the animal experiments (approval no. 2020-10-002).

\section{Patient consent for publication}

Not applicable.

\section{Competing interests}

The authors declare that they have no competing interests.

\section{References}

1. Guan Q: A Comprehensive review and update on the pathogenesis of inflammatory bowel disease. J Immunol Res: Dec 1, 2019 (Epub ahead of print). doi: 10.1155/2019/7247238.

2. Desreumaux P and Colombel JF: Modifications and roles of intestinal flora in inflammatory bowel diseases. Gastroenterol Clin Biol 25: C89-C93, 2001.

3. Huang Y and Chen Z: Inflammatory bowel disease related innate immunity and adaptive immunity. Am J Transl Res 8: 2490-2497, 2016.

4. Coskun M, Vermeire S and Nielsen OH: Novel targeted therapies for inflammatory bowel disease. Trends Pharmacol Sci 38: 127-142, 2017.

5. Rogler G: Where are we heading to in pharmacological IBD therapy? Pharmacol Res 100: 220-227, 2015.

6. Zheng K, Shen H, Jia J, Lu Y, Zhu L, Zhang L and Shen Z: Traditional Chinese medicine combination therapy for patients with steroid-dependent ulcerative colitis: Study protocol for a randomized controlled trial. Trials 18: 8, 2017.

7. Sałaga M,Zatorski H, Sobczak M, Chen C and Fichna J: Chinese herbal medicines in the treatment of IBD and colorectal cancer: A review. Curr Treat Options Oncol 15: 405-420, 2014.

8. Shicai H, Kang D and Xu Y: Clinical efficacy of Kuijie enema liquid by air- ressured herb enema combined with Zhuling Xianglian decoction in the treatment of ulcerative colitis and its effect on the level of inflammatory factors of patients. Hebei J Tradit Chin Med 41: 367-371, 2019 (In Chinese).

9. Shen T, He YL, Sun GP, Liu WX and Zheng SZ: Studies on chemical constituents of Sanguisorba longifolia Bertol. Indian J Chem B 47: 1600-1604, 2008.

10. He X, Wang X, Fang J, Zhao Z, Huang L, Guo H and Zheng X: Bletilla striata: Medicinal uses, phytochemistry and pharmacological activities. J Ethnopharmacol 195: 20-38, 2017.

11. Feng S, Dai Z, Liu A, Wang H, Chen J, Luo Z and Yang CS: $\beta$-Sitosterol and stigmasterol ameliorate dextran sulfate sodium-induced colitis in mice fed a high fat Western-style diet. Food Funct 8: 4179-4186, 2017.

12. Paniagua-Pérez R, Flores-Mondragón G, Reyes-Legorreta C, Herrera-López B, Cervantes-Hernández I, Madrigal-Santillán O, Morales-González JA, Álvarez-González I and MadrigalBujaidar E: Evaluation of the anti-inflammatory capacity of beta-sitosterol in rodent assays. Afr J Tradit Complement Altern Med 14: 123-130, 2016. 
13. Liz R, Zanatta L, dos Reis GO, Horst H, Pizzolatti MG, Silva FR and Fröde TS: Acute effect of $\beta$-sitosterol on calcium uptake mediates anti-inflammatory effect in murine activated neutrophils. J Pharm Pharmacol 65: 115-122, 2013.

14. Kim KA, Lee IA, Gu W, Hyam SR and Kim DH: $\beta$-Sitostero attenuates high-fat diet-induced intestinal inflammation in mice by inhibiting the binding of lipopolysaccharide to toll-like receptor 4 in the NF- $\mathrm{KB}$ pathway. Mol Nutr Food Res 58: 963-972, 2014

15. Bin Sayeed MS, Karim SMR, Sharmin T and Morshed MM: Critical analysis on characterization, systemic effect, and therapeutic potential of beta-sitosterol: A plant-derived orphan phytosterol. Medicines (Basel) 3: 3, 2016.

16. Yin Y, Liu X, Liu J, Cai E, Zhu H, Li H, Zhang L, Li P and Zhao Y: Beta-sitosterol and its derivatives repress lipopolysaccharide/d-galactosamine-induced acute hepatic injury by inhibiting the oxidation and inflammation in mice. Bioorg Med Chem Lett 28: 1525-1533, 2018.

17. Simin F, Ke N, Ping S, Guoping R, Peilong S and Zisheng L: Research on the $\beta$-sitosterol and stigmasterol therapeutic effect of acute colitis in mice. J Chin Cereals Oils Assoc 33: 80-86, 94, 2018

18. Fraile L, Crisci E, Córdoba L, Navarro MA, Osada J and Montoya M: Immunomodulatory properties of beta-sitosterol in pig immune responses. Int Immunopharmacol 13: 316-321, 2012

19. Wirtz S, Neufert C, Weigmann B and Neurath MF: Chemically induced mouse models of intestinal inflammation. Nat Protoc 2 : 541-546, 2007

20. Ramadass SK, Jabaris SL, Perumal RK, HairulIslam VI, Gopinath A and Madhan B: Type I collagen and its daughter peptides for targeting mucosal healing in ulcerative colitis: A new treatment strategy. Eur J Pharm Sci 91: 216-224, 2016.

21. Xia Z, Han Y, Wang K, Guo S, Wu D, Huang X, Li Z and Zhu L: Oral administration of propionic acid during lactation enhances the colonic barrier function. Lipids Health Dis 16: 62, 2017

22. Tao JH, Duan JA, Jiang S, Guo JM, Qian YY and Qian DW: Simultaneous determination of six short-chain fatty acids in colonic contents of colitis mice after oral administration of polysaccharides from Chrysanthemum morifolium Ramat by gas chromatography with flame ionization detector. J Chromatogr B Analyt Technol Biomed Life Sci 1029-1030: 88-94, 2016.

23. Patel A, Panchal H and Dubinsky MC: Fecal calprotectin levels predict histological healing in ulcerative colitis. Inflamm Bowe Dis 23: 1600-1604, 2017.

24. Okayasu I, Hatakeyama S, Yamada M, Ohkusa T, Inagaki Y and Nakaya R: A novel method in the induction of reliable experimental acute and chronic ulcerative colitis in mice. Gastroenterology 98: 694-702, 1990.

25. Gisbert JP and McNicholl AG: Questions and answers on the role of faecal calprotectin as a biological marker in inflammatory bowel disease. Dig Liver Dis 41: 56-66, 2009.

26. Erbayrak M, Turkay C, Eraslan E, Cetinkaya H, Kasapoglu B and Bektas M: The role of fecal calprotectin in investigating inflammatory bowel diseases. Clinics (São Paulo) 64: 421-425, 2009.

27. Zha Z, Lv Y, Tang H, Li T, Miao Y, Cheng J, Wang G, Tan Y, Zhu Y, Xing X, et al: An orally administered butyrate-releasing xylan derivative reduces inflammation in dextran sulphate sodium-induced murine colitis. Int J Biol Macromol 156 1217-1233, 2020.

28. Gao X, Cao Q, Cheng Y, Zhao D, Wang Z, Yang H, Wu Q, You L, Wang Y, Lin Y, et al: Chronic stress promotes colitis by disturbing the gut microbiota and triggering immune system response. Proc Natl Acad Sci USA 115: E2960-E2969, 2018.
29. Chang PV, Hao L, Offermanns S and Medzhitov R: The microbial metabolite butyrate regulates intestinal macrophage function via histone deacetylase inhibition. Proc Natl Acad Sci USA 111: 2247-2252, 2014

30. Yan JB, Luo MM, Chen ZY and He BH: The function and role of the Th17/Treg cell balance in inflammatory bowel disease. J Immunol Res: Dec 15, 2020 (Epub ahead of print). doi: 10.1155/2020/8813558.

31. Hansberry DR, Shah K, Agarwal P and Agarwal N: Fecal myeloperoxidase as a biomarker for inflammatory bowel disease. Cureus 9: e1004-e1004, 2017.

32. Huang JH, Huang XH, Chen ZY and Zheng QS: Dose conversion among different animals and healthy volunteers in pharmacological study. Chinese J Pharmacol Toxicol 9: 1069-1072, 2004.

33. Wlodarska M, Kostic AD and Xavier RJ: An integrative view of microbiome-host interactions in inflammatory bowel diseases. Cell Host Microbe 17: 577-591, 2015.

34. Zhu ZH, Wang QY and Wu Q: On the examination of the Darcy permeability of soft fibrous porous media; new correlations. Chem Eng Sci 173: 525-536, 2017.

35. Bamias G, Pizarro TT and Cominelli F: Pathway-based approaches to the treatment of inflammatory bowel disease. Transl Res 167: 104-115, 2016.

36. Eck A, Zintgraf LM, de Groot EF, de Meij TG, Cohen TS, Savelkoul PH, Welling $M$ and Budding AE: Interpretation of microbiota-based diagnostics by explaining individual classifier decisions. BMC Bioinformatics 18: 441, 2017.

37. Feng Y, Wang Y, Wang P, Huang Y and Wang F: short-chain fatty acids manifest stimulative and protective effects on intestinal barrier function through the inhibition of NLRP3 inflammasome and autophagy. Cell Physiol Biochem 49: 190-205, 2018.

38. Tian Y, Xu Q, Sun L, Ye Y and Ji G: Short-chain fatty acids administration is protective in colitis-associated colorectal cancer development. J Nutr Biochem 57: 103-109, 2018.

39. Levy M,Thaiss CA,ZeeviD,DohnalováL,Zilberman-SchapiraG, Mahdi JA, David E, Savidor A, Korem T, Herzig Y, et al: Microbiota-modulated metabolites shape the intestinal microenvironment by regulating NLRP6 inflammasome signaling. Cell 163: 1428-1443, 2015.

40. Zhang SL, Wang SN and Miao CY: Influence of microbiota on intestinal immune system in ulcerative colitis and its intervention. Front Immunol 8: 1674, 2017.

41. Ahmadi M, Yousefi M, Abbaspour-Aghdam S, Dolati S, Aghebati-Maleki L, Eghbal-Fard S, Khabbazi A, Rostamzadeh D, Alipour S, Shabani M, et al: Disturbed Th17/Treg balance, cytokines, and miRNAs in peripheral blood of patients with Behcet's disease. J Cell Physiol 234: 3985-3994, 2019.

42. Gálvez J: Role of Th17 cells in the pathogenesis of human IBD. ISRN Inflamm 2014: 928461, 2014.

43. Miossec P and Kolls JK: Targeting IL-17 and TH17 cells in chronic inflammation. Nat Rev Drug Discov 11: 763-776, 2012.

44. Hus I, Maciag E and Roliński J: The role of Th17 cells in anti-cancer immunity. Postepy Hig Med Dosw 64: 244-250, 2010 (In Polish).

45. Bettelli E, Carrier Y, Gao W, Korn T, Strom TB, Oukka M, Weiner HL and Kuchroo VK: Reciprocal developmental pathways for the generation of pathogenic effector TH17 and regulatory T cells. Nature 441: 235-238, 2006.

This work is licensed under a Creative Commons Attribution-NonCommercial-NoDerivatives 4.0 International (CC BY-NC-ND 4.0) License. 